Resolución endovascular de pseudoaneurisma poplíteo gigante

\title{
Endovascular resolution of giant popliteal pseudoaneurysm
}


Resolución endovascular de pseudoaneurisma poplíteo gigante Endovascular resolution of giant popliteal pseudoaneurysm

Diego Ardiles López ${ }^{1}$, Martín Alanís Álvarez², Manuel Santander Hess³ Rocío Castro Venegas ${ }^{4}$, Sergio Collao Zlosilo ${ }^{4}$

${ }^{1}$ Servicio de Cirugía. Hospital Dr. Hernán Henríquez Aravena. Temuco, Chile. ${ }^{2}$ Servicio de Cirugía. Clínica RedSalud Mayor. Temuco, Chile. ${ }^{3}$ Departamento de Cirugía Torácica y Cardiovascular. Universitätsmedizin Göttingen. Gottingen, Alemania. ${ }^{4}$ CESFAM Nueva Imperial. Servicio de Salud Araucanía Sur. Nueva Imperial, Chile. ${ }^{5}$ Servicio Médico Quirúrgico. Dr. Hospital Víctor Ríos Ruiz. Los Ángeles, Chile

Recibido: 31/08/2021

Aceptado: 02/09/2021

Correspondencia: Diego Ardiles López. Servicio de Cirugía. Hospital Dr. Hernán Henríquez Aravena. Manuel Montt, 115. Temuco, Chile e-mail: diego.ardiles.l@gmail.com

DOI: 10.20960/angiologia.00349

Conflictos de interés: los autores declaran no tener ningún conflicto de interés.

\section{RESUMEN}


Introducción: el pseudoaneurisma poplíteo es una entidad infrecuente y suele ser secundario a un trauma. Se presenta el caso de pseudoaneurisma poplíteo traumático.

Caso clínico: hombre de 74 años con fractura de fémur hace 4 meses, manejada con implantes externos antes de la resolución definitiva. Presentó sangrado local y aumento del volumen de la extremidad inferior izquierda. El angio-TAC evidenció un gran pseudoaneurisma de la arteria poplítea izquierda que se resolvió de manera endovascular con stent cubierto.

Discusión: el tratamiento endovascular es una opción para la resolución del pseudoaneurisma poplíteo. El tratamiento quirúrgico abierto debe considerarse en casos de inestabilidad hemodinámica y la posible coexistencia de otras lesiones que requieran manejo inmediato.

Palabras clave: Pseudoaneurisma. Stents. Trauma.

\section{ABSTRACT}

Introduction: popliteal pseudoaneurysm is a rare entity and is usually secondary to trauma. A case of traumatic popliteal pseudoaneurysm is presented.

Clinical case: a 74-year-old man with a femur fracture 4 months ago, managed with external tutors prior to final resolution. He presented local bleeding and increased volume of the left lower limb. AngioTC showed a large pseudoaneurysm of the left popliteal artery. We resolve it with endovascular treatment with a covered stent.

Discussion: endovascular treatment is an option for the resolution of popliteal pseudoaneurysm. Open surgical treatment should be considered in cases of hemodynamic instability and the possible coexistence of other injuries that require immediate management.

Keywords: Pseudoaneurysm. Stents. Trauma. 


\section{INTRODUCCIÓN}

El pseudoaneurisma poplíteo es una entidad poco frecuente y suele estar asociada a eventos traumáticos o a lesiones iatrogénicas $(1,2)$. Se presenta el caso de un paciente de 74 años con pseudoaneurisma de la arteria poplítea posterior a una fractura de fémur y a una cirugía traumatológica de urgencia.

\section{CASO CLÍNICO}

Hombre de 74 años con antecedentes de HTA que se fracturó el fémur y la tibia de la pierna izquierda por caída mientras montaba a caballo hace 2 meses. Se manejó con implantes externos a la espera de la resolución definitiva de su fractura. Presentó sangrado reciente desde el sitio de inserción de los implantes con aumento de volumen del muslo y de la pierna izquierda, por lo que acudió al Servicio de Urgencias. Se realizó un angio-TAC de las extremidades inferiores que evidenció un gran pseudoaneurisma de $70 \times 55 \mathrm{~mm}$ en sus ejes mayores dependiente del segundo segmento de la arteria poplítea izquierda (Fig. 1). El sangrado se controló con compresión local y el paciente se mantuvo estable, sin caída significativa de su hematocrito. Un dúplex corroboró la permeabilidad del pseudoaneurisma y destacó, además, una trombosis venosa femoropoplítea de la misma extremidad.

Se consideró la cirugía abierta para el drenaje del hematoma local y la reparación del pseudoaneurisma como primera alternativa, pero se descartó ante la imposibilidad de acceder quirúrgicamente a la arteria poplítea debido a la presencia de los implantes externos a lo largo de toda la extremidad. Se decidió no anticoagular al paciente debido al riesgo de hemorragia y se instaló un filtro de vena cava inferior Celect $^{\mathrm{Tm}}$ de Cook. Se realizó angiografía selectiva por sustracción digital por punción femoral derecha, se avanzó vaina Destination ${ }^{\circledR}$ 8F de Terumo ${ }^{\circledR}$ 
sobre guía Amplatz 0,035" de Cencomex y se desplegó stent cubierto Viabahn ${ }^{\circledR} 8 \times 50 \mathrm{~mm}$ de Gore ${ }^{\circledR}$ para lograr exclusión del pseudoaneurisma (Fig. 2).

Evolución favorable, sin dolor ni nuevos eventos de sangrado, sin isquemia de la extremidad. Regresión lenta del edema durante la hospitalización. Se optó por doble antiagregación (ácido acetilsalicílico + clopidogrel) sobre terapia triple (ácido acetilsalicílico + clopidogrel + anticoagulación) para el manejo posoperatorio y se decidió el alta dada la remisión de los síntomas.

En el control a los 30 días, el paciente se mantuvo asintomático, sin edema ni dolor de la extremidad, y el dúplex demostró la exclusión del pseudoaneurisma y la regresión parcial del hematoma, además de signos de repermeabilización del sistema venoso profundo.

\section{DISCUSIÓN}

El trauma de la arteria poplítea es de los traumas arteriales periféricos más serios, ya que conlleva un riesgo significativo de complicaciones y pérdida de la extremidad. El pseudoaneurisma poplíteo suele estar causado por la laceración de la arteria y está asociado en un $62 \%$ de las ocasiones a lesiones traumáticas penetrantes y en un $37 \%$, a lesiones iatrogénicas (1-3). Su presentación suele ser tardía y los hallazgos clínicos más comunes son el edema doloroso y una masa pulsátil al examen físico. La sospecha clínica debe confirmarse con un estudio de imagen acabado mediante angio-TAC y angiografía por sustracción digital para identificar la lesión y planificar la cirugía $(4,5)$.

Respecto a su tratamiento, no existe evidencia que demuestre la superioridad de la elección endovascular y la mayoría se trata con cirugía abierta (5-7). El tratamiento endovascular con stent cubierto es una opción factible, siempre mediada por una adecuada planificación, identificando las zonas de despliegue y las colaterales del segmento objetivo, que pueden mantener la permeabilidad de la lesión pese a una 
adecuada cobertura. Debe considerarse la resolución quirúrgica abierta en casos de inestabilidad hemodinámica y la posible coexistencia de otras lesiones que requieran manejo inmediato; sin embargo, el abordaje suele ser difícil, con necesidad de grandes incisiones (6-9). En este caso en particular, debido a la antigüedad de la lesión, a la estabilidad del paciente y a la presencia de implantes externos que dificultaban la exposición, la terapia endovascular fue la opción más adecuada.

La presencia de trombosis venosa profunda es un evento de carácter multifactorial. En el presente caso, el trauma, el reposo prolongado por la inmovilización y los factores locales dados principalmente por la compresión del gran pseudoaneurisma generaron el ambiente propicio para su desarrollo. La elección de la anticoagulación frente a la terapia doble antiagregante o terapia triple plantea un escenario complejo en situaciones como esta. Debe considerarse la clínica del paciente, el riesgo de hemorragia y los beneficios a largo plazo propios de la doble antiagregación en la permeabilidad del stent (10).

\section{BIBLIOGRAFÍA}

1. Megalopoulos A, Siminas S, Trelopoulos G. Traumatic Pseudoaneurysm of the Popliteal Artery After Blunt Trauma: Case Report and a Review of the Literature. Vasc Endovasc Surg 2007;40(6):499-504. DOI: 10.1177/1538574406290037

2. Oliveira GP, Guillaumon AT, De Brito IB, Lima JMT, Benvindo SC, dos Santos LG. Pseudoaneurisma idiopático da artéria poplítea: abordagem diagnóstico-terapêutica na urgencia. J Vasc Bras 2014;13:244-8. DOI: 10.1590/jvb.2014.032

3. Raherinantenaina F, Rajaonanahary TM, Rakoto HR. Management of popliteal artery pseudoaneurysms as a result of limb trauma and orthopedic surgery or associated with osteochondromas. Ann 
Cardiol

Angeiol

$2016 ; 65: 265-74$

DOI:

10.1016/j.ancard.2016.04.023

4. Khalifeh A, Kantar R, Watson JDB, Cox S, Sarkar R, Toursvadakohi S. Management of chronic massive traumatic popliteal artery pseudoaneurysm: a case series with different treatment strategies. Vasc Endovascular Surg 2018;52:75-9. DOI: $10.1177 / 1538574417739748$

5. Nogueira ACS, Salgdo CG, Nogueira FBS, Amaral SI, Rabischoffsky A. Pseudoaneurismas: quando E como tratálos. Arq Bras Cardiol 2013;26:289-307.

6. Matsushita M, Nishikimi N, Sakurai T, Nimura Y. Pseudoaneurysm of the popliteal artery caused by exostosis of the femur: case report and review of the literature. J Vasc Surg 2000;32:201-4. DOI: $10.1067 / \mathrm{mva} .2000 .105679$

7. Ohrlander T, Holst J, Malina M. Emergency intervention for thrombosed popliteal artery aneurysm: can the limb be salvaged? J Cardiovasc Surg 2007;48(3):289-97.

8. Gillespie DL, Cantelmo NL. Traumatic popliteal artery pseudoaneurysms: case report and review of the literature. J Trauma 1991;31(3):412-5. DOI: 10.1097/00005373-19910300000019

9. Pérez-Burkhardt JL, Gómez Castilla JC. Postraumatic popliteal pseudoaneurysm from femoral osteochondroma: case report and review of the literature. J Vasc Surg 2003;37:669-71. DOI: $10.1067 / \mathrm{mva} .2003 .48$

10. Singh P, Harper Y, Oliphant CS, Morsy M, Skelton M, Askari R, et al. Peripheral interventions and antiplatelet therapy: Role in current practice. World J Cardiol 2017;9(7):583-93. DOI: 10.4330/wjc.v9.i7.583 


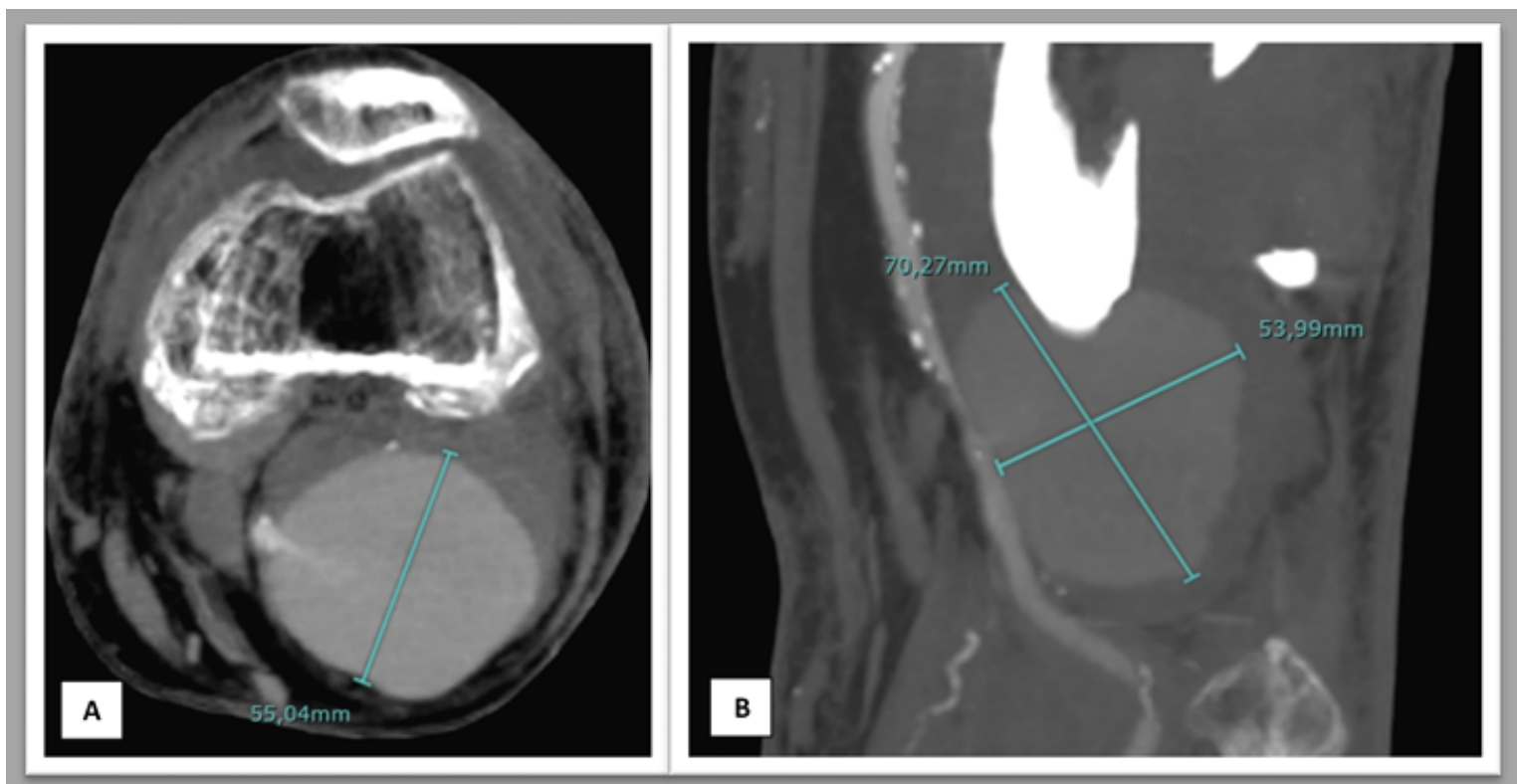

Figura 1. Angio-TAC de extremidad inferior izquierda con cortes axial (A) y coronal (B) a nivel del hueco poplíteo. Se evidencia gran pseudoaneurisma con fuga activa de contraste y dependiente de la segunda porción de la arteria poplítea izquierda.

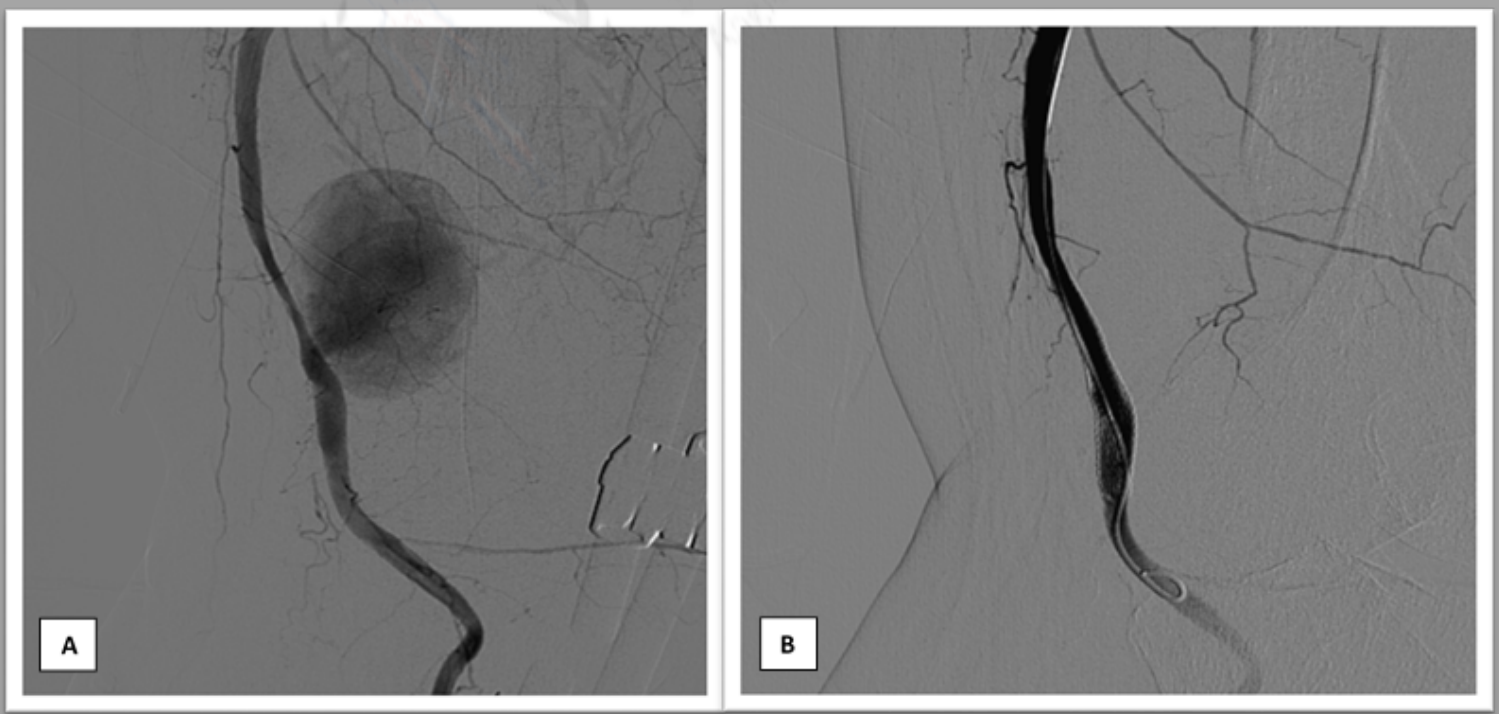

Figura 2. Angiografía por sustracción digital de la extremidad inferior izquierda. A. Gran pseudoaneurisma dependiente de la segunda porción de la arteria poplítea. B. Exclusión completa del pseudoaneurisma después del despliegue de stent cubierto. 


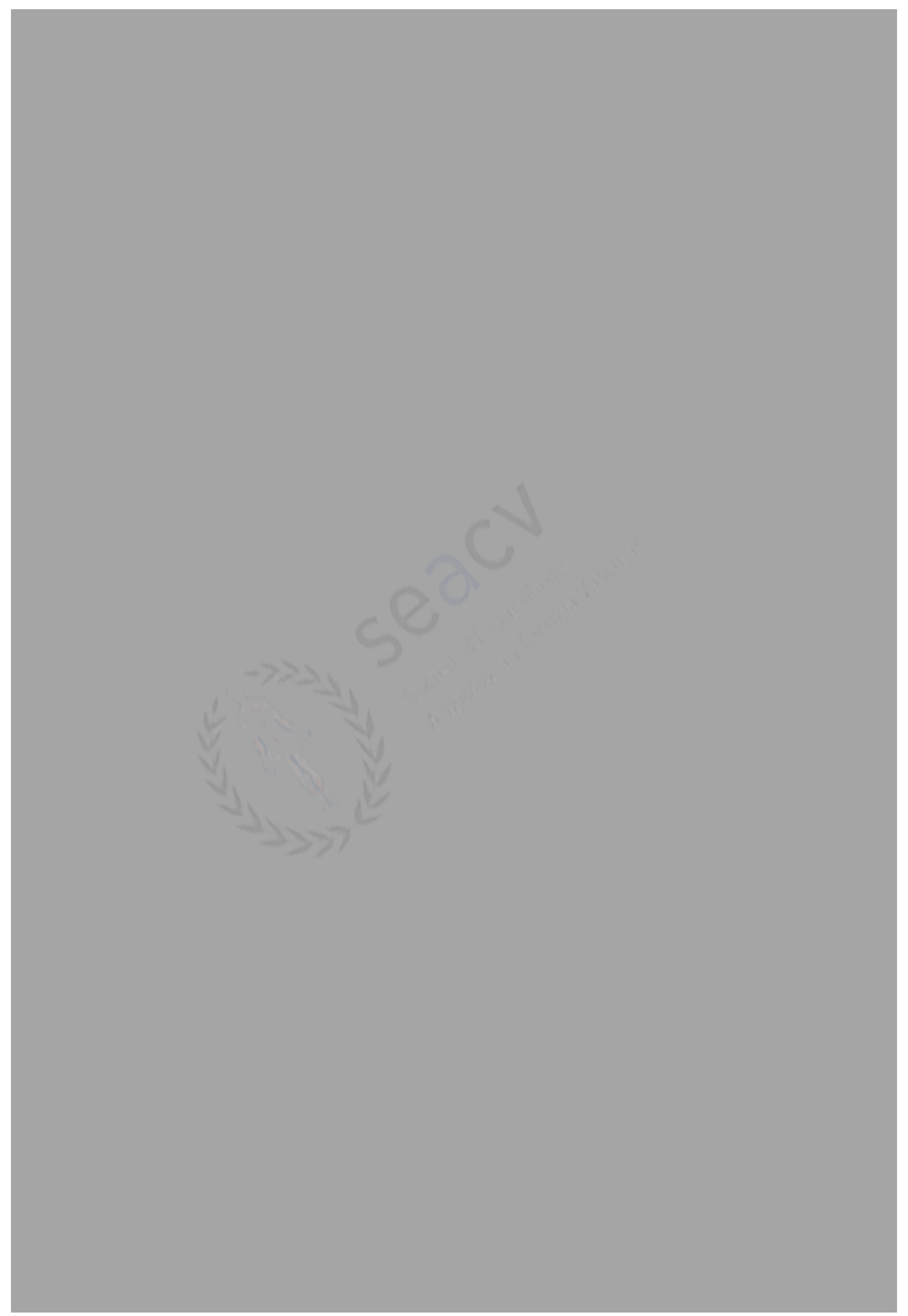

